\title{
Investigations of Structural Behavior According to Frame Shape Variation of Electric Transmission Tower
}

\author{
Woo Bum Kim
}

\begin{abstract}
The purpose of this study is to analyze the ultimate strength and behavior of triangular frame and rectangular frame in elastic transmission tower. Investigation of collapse mechanism including local and global failure of partial frame was carried out through nonlinear finite element analysis . Ultimate strength and deformation was investigated in the case of shape variation by changing the inner and outer frame. The necessity of rectangular frame reducing sub-brace member was suggested through comparing the various combination of frame shape.
\end{abstract}

Index Terms-Transmission tower, triangular frame, rectangular frame, ultimate load capacity, nonlinear analysis, finite element model.

\section{INTRODUCTION}

The frame shape in electric transmission tower is very important in safety and economic feasibility. However, due to complexity in the behavior, traditional conservative design practice[1]. based on truss analysis has still been adopted.

Truss structure in principle is in triangular shape. Rectangular shape that causes instability has been excluded from the design. The triangular shape frame is stable in structural behavior, however it results in excessive sub-brace in the frame. Actually, the post that serves the main member in transmission tower is erected as continuous member, and the brace is fixed to main member by plate welding. Thus, transmission behaves rather similar to frame behavior that resists to the moment than truss behavior. Thus, frame analysis through 3D frame nonlinear analysis is more reasonable to predict the structural behavior and maximum load capacity of transmission tower[2]-[4].

Load capacity of the frame forming the transmission tower depends on the characteristics of unit member and the shape of the frame comprising of the members[5]. However, a very few studies on deformation patterns of the frame have been conducted because of geometric complexity of the frame. Behavioral characteristics of 2D frame are different with that of $3 \mathrm{D}$ frame. Because of buckling behavior due to movement of joint, plastic deformation and redistribution of stress, general analytical method does not gives accurate results. Thus, analytical model that could incorporate such characteristics is required and verification is needed.

Hence, this study is intended to identify the role of the

Manuscript received November 9, 2017; revised May 1, 2018.

Woobum Kim is with the Kongju National University, Korea (e-mail: wbk@kongju.ac.kr). sub-braces and the characteristics of frame behavior through 3D nonlinear finite element analysis. Comparative verification so as to come up with the basis to minimize the sub-braces and maximize cost efficiency of transmission tower is performed.

\section{STRUCTURAL ANALYSIS}

The method to estimate the buckling load based on theoretical analysis may consider the effect of joint restraint in plane[6]. However, The method is not applicable to three dimensional frame which shows out of plane joint movement. Also, it is difficult to estimate the inelastic buckling load capacity by theoretical approach when plastic deformation occurs in joint at the end[7]. Thus finite element model that considers such deformation was developed and nonlinear analysis was performed. In this study, when leg part of transmission tower (hereinafter "leg") is built with triangular frame or rectangular frame, behavior and load capacity of the both frame were compared.

\section{A. Finite Element Analysis}

As the load capacity of the leg is greatly dependent on variation of local stress of the joint, it's difficult to evaluate the load capacity with theoretical approach. Thus, to estimate the load capacity of the leg, finite element analysis for the leg was carried out. Analysis model for the leg was based on shape of $765 \mathrm{kV}$ transmission tower. Finite element analysis was conducted using ABAQUS 6.12.[8] S4R5 Shell element was adopted to consider local stress distribution and plastic deformation of the member and joint. This is quadrilateral shell element with 5 degrees of freedom at each joint. A truss analysis so far is difficult to accurately incorporate the redistribution of stress and also has many problems in identifying the buckling behavior of the member because of the analysis assuming the joint as a pin. Thus to incorporate the material and geometric nonlinear behavior of the structure, element discretization was made every $1 / 10$ of diameter of member. In this finite element analysis, first, accurate buckling load capacity and buckling mode of the member at extreme status were compared with actual structural test result and second, characteristics of buckling behavior of rectangular frame introduced was compared with the triangular frame. Thus, finite element analysis model of the leg with triangular and rectangular frame was developed and as shown in Fig 1, and lateral load was applied to investigate the capacity and failure shape of the frame.

\section{1) Deformation shape of the frame}

Buckling load capacity is dependent on buckling mode and thus buckling load capacity can be compared from 
buckling deformation shape. Fig. 2 shows buckling deformation of triangular and rectangular frame obtained from the finite element analysis. Triangular frame is what applied to existing transmission tower while rectangular frame is what proposed in this study. As shown in Fig 2, there's a difference between triangular frame and rectangular frame.

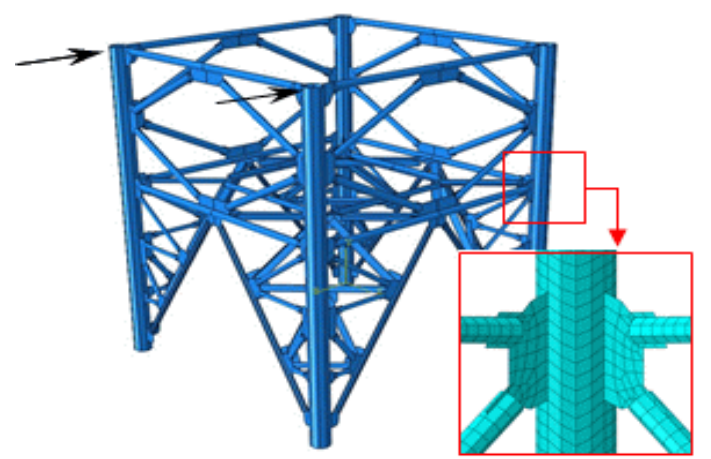

Fig. 1. Finite element analysis model and loading of leg part in transmission tower

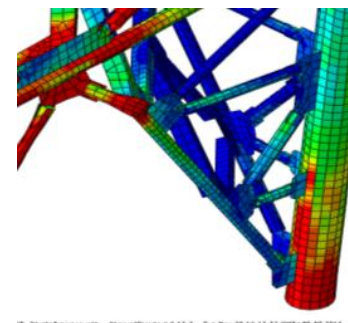

Birts

(a) Triangular frame

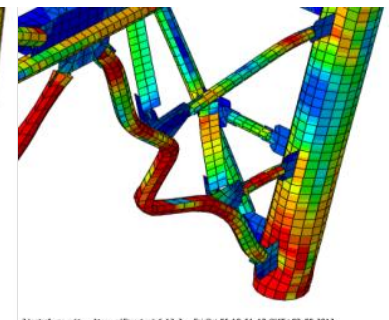

$\Rightarrow \mathrm{Ary}$

(b) Rectangular frame
Fig. 2. Deformation shape of triangular/rectangular frame.

In case of triangular frame as shown in Fig. 2 (a), after an out-of-plane buckling occurred on the first joint on top of brace, it reached to ultimate load capacity. This is a typical failure pattern occurred in one member constrained at both ends. The buckling was not in-plane buckling but out-ofplane buckling. This is due to the fact that the rotational stiffness at the joint in out-of-plane direction is less than that of in-plane direction. In case of a rectangular frame, Overall buckling occurred over entire brace (3member) as shown in Fig. 2 (b). Overall buckling was out of plane buckling same as shown in triangular frame. The reason that out of plane buckling governs in both cases is because of joint movement of out of plane direction. It is inferred that joint movement effect considering 3 dimensional direction should be considered to estimate the accurate buckling strength. Therefore, it is needed that leg of transmission tower should be analyzed by three dimensional model.

\section{2) Behavioral characteristics of frame}

As seen in Fig. 3, stiffness of rectangular frame is less than triangular frame which was attributable to reduced overall stiffness of the structure due to removal of subbraces. As seen in Table I, initial stiffness of triangular frame in elastic zone was higher than rectangular frame by $14 \%$. And in terms of ultimate load capacity, triangular frame was higher than rectangular frame by $0.4 \%$.
Displacement by plastic deformation in rectangular frame was greater than triangular frame and the difference in displacement at the ultimate load capacity reached to $84 \%$. Thus initial stiffness and load capacity was dependent on sub-braces. Difference in maximum load capacity between triangular frame and rectangular frame was not significant, while, deformation was significant and additional consideration for the deformation is required. However, when using the allowable stress design concept adopted in current design, difference between triangular frame and rectangular frame is insignificant.

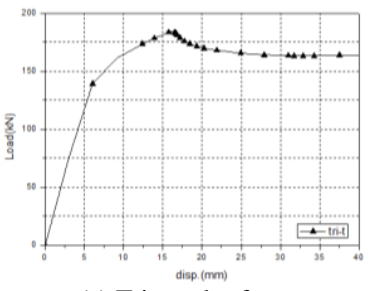

(a) Triangular frame

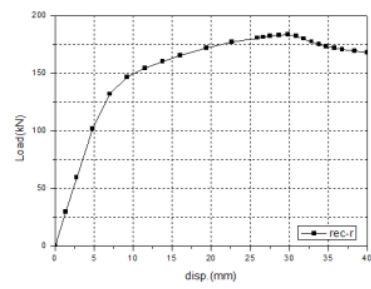

(b) Rectangular frame
Fig. 3. Load capacity of triangular/rectangular frame.

TABLE I: ANALYSIS RESULT

\begin{tabular}{|l|c|c|c|}
\hline \multirow{2}{*}{$\begin{array}{l}\text { Triangular } \\
\text { frame }\end{array}$} & $\begin{array}{c}\text { Initial stiffness } \\
(\mathrm{N} / \mathrm{mm})\end{array}$ & $\begin{array}{c}\text { Ultimate load } \\
(\mathrm{kN})\end{array}$ & $\begin{array}{l}\text { Displacement at } \\
\text { ultimate load }(\mathrm{mm})\end{array}$ \\
\cline { 2 - 4 } & 24798 & 184 & 16.1 \\
\hline $\begin{array}{l}\text { Rect angular } \\
\text { frame }\end{array}$ & 21686 & 183 & 29.8 \\
\hline
\end{tabular}

\section{THE EFFECT OF LEG SHAPE ON LOAD CAPACITY OF THE TOWER}

The effect of Leg shape on load capacity was investigated. The leg frame in 3D shape is divided into front frame and inner frame and the variation of load capacity while combining the front frame and inner frame in different shape was monitored, and the possibility of removing the subbraces was examined based on the result.

\section{A. Type of Analysis Model and Frame}

Combination of the frame by type is as Table II. Model parameters included existing triangular frame of transmission, rectangular frame with reduced number of members, triangular frame with extended internode and rectangular frame with extended internode. The frame on leg id divided into outer frame and inner frame. The frame is divided into triangular frame and rectangular frame as shown in Table III.

TABLE II: TYPE OF ANALYSIS MODEL

\begin{tabular}{c|c|c|c|c}
\hline inner & tri & rec & tri_long & rec_long \\
\hline Tri & tri-t & tri-r & tri-tl & tri-rl \\
\hline Rec & rec-t & rec-r & rec-tl & rec-rl \\
\hline
\end{tabular}




\begin{tabular}{c|c|c|c|c}
\cline { 2 - 4 } tri_long & tri_long-t & tri_long-r & tri_long-tl & tri_long-rl \\
\hline rec_long & rec_long-t & rec_long-r & rec_long-tl & rec_long-rl \\
\hline
\end{tabular}

\section{B. Frame Analysis}

Finite element analysis was conducted using the same model used previously and the analysis was conducted with 16 types of model by combining 4 front frames and 4 inner frames.

\section{1) Deformation of the frame}

As shown in Fig. 4(a), buckling occurred on top of the brace receiving the compressive force in case of triangular front and inner frame and in such a case, effective buckling length is considered the length on top, not the whole length of the brace. And it was the out-of-the plane buckling, instead of in-plane buckling, which is attributable to joint movement .

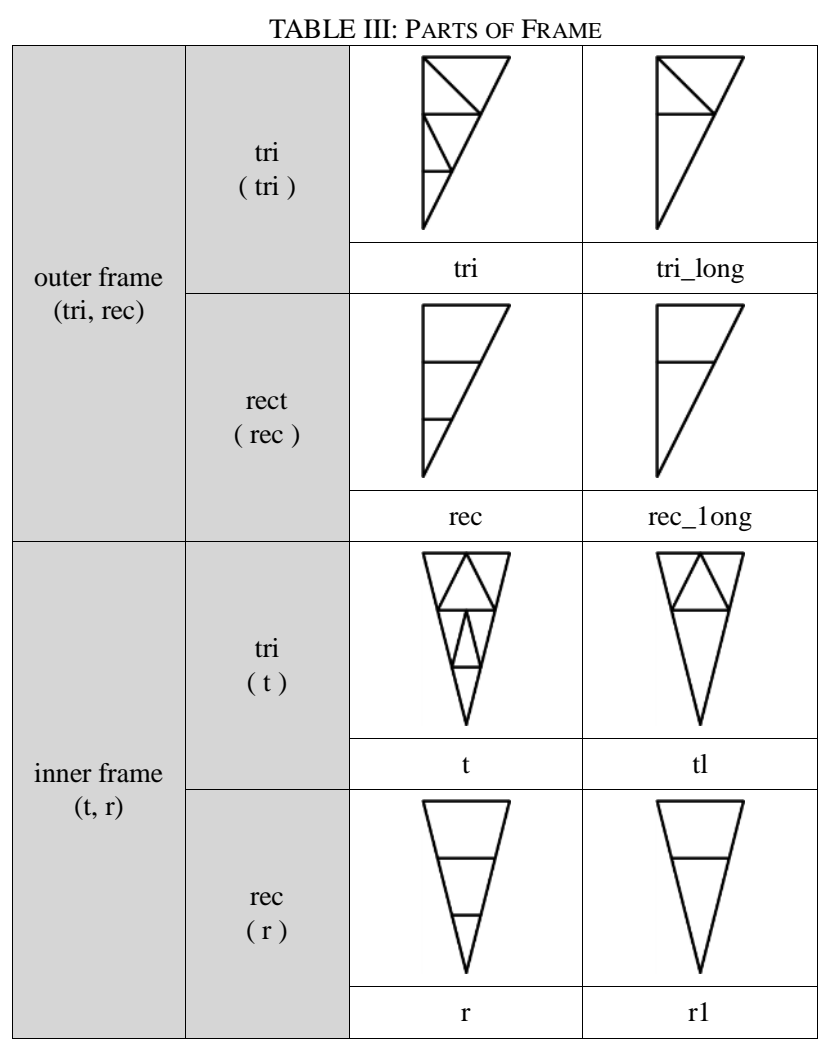

When front or inner frame is rectangular frame as Fig. 4(b), buckling occurred over entire part of the brace which was attributable to different deformation shape due to varying constraint after removing the sub-braces.

Viewing Fig. 4(c) and Fig. 4(d), when a front frame and inner frame is tri-long and rec-long, deformation due to increase in buckling length appeared.

\section{2) Behavioral characteristics of the frame}

Behavioral characteristics was compared and evaluated after classifying the front frame into 4 types such as tri, rec, tri-long and rec-long and classifying the inner frame into 4 types such as $\mathrm{t}, \mathrm{r}, \mathrm{tl}$ and $\mathrm{rl}$ and combining the front and inner frame. The result from comparing the load-displacement characteristics while varying the inner frame based on front frame is as follows.

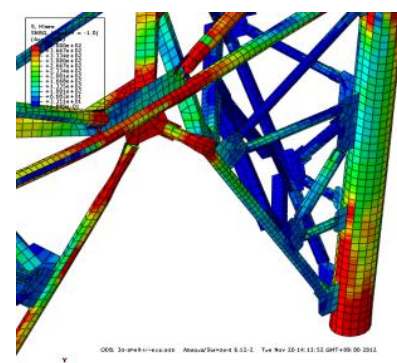

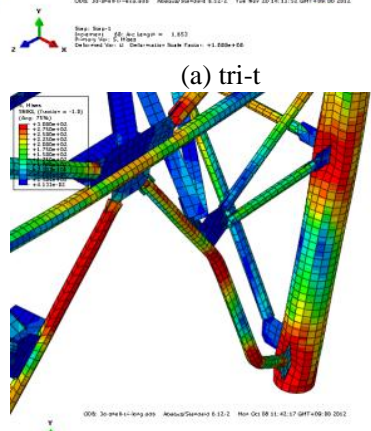

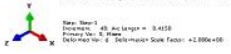

(c) tri_long-tl

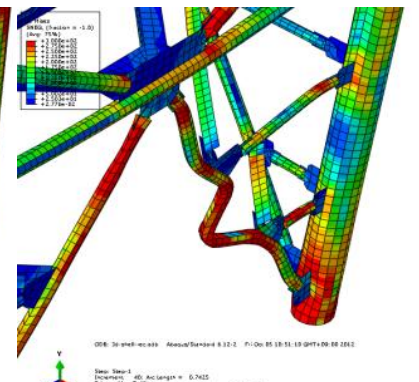

人.

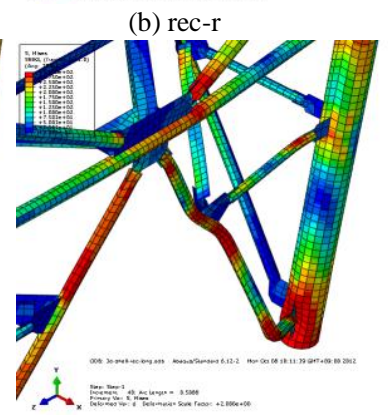

(d) rec_long-rl
Fig. 4. Typical model deformation shapes

\section{3) Outer triangular frame}

Fig. 5 shows the graph comparing the load-deformation curve with outer frame in the type of triangular frame and 4 types of inner frame such as $\mathrm{t}, \mathrm{r}, \mathrm{tl}$ and $\mathrm{rl}$. As seen in graph, 4 frames indicated a similar initial stiffness but a different maximum load capacities.

When inner frame is triangular frame (tri-t) or rectangular frame, load-deformation curve was similar proving that a horizontal sub-braces on inner frame alone has the effect of constraining the movement of the joint. But in case of triangular frame (tri-tl,tri-rl) with extended inner frame, maximum load capacity was less than a triangular frame (trit,tri-r) by $6 \%$ which was attributable to reduced buckling load capacity due to increase in buckling length on brace.

\section{4) Outer rectangular frame}

Fig. 6 shows the graph comparing the load-deformation curve with a front frame in the type of rectangular frame and 4 types of inner frame such as $\mathrm{t}, \mathrm{r}, \mathrm{tl}$ and $\mathrm{rl}$ and as seen in the graph, 4 frames indicated a similar initial stiffness but the maximum load capacity was reduced in case of a triangular frame (tri-tl,tri-rl) with extended inner frame.

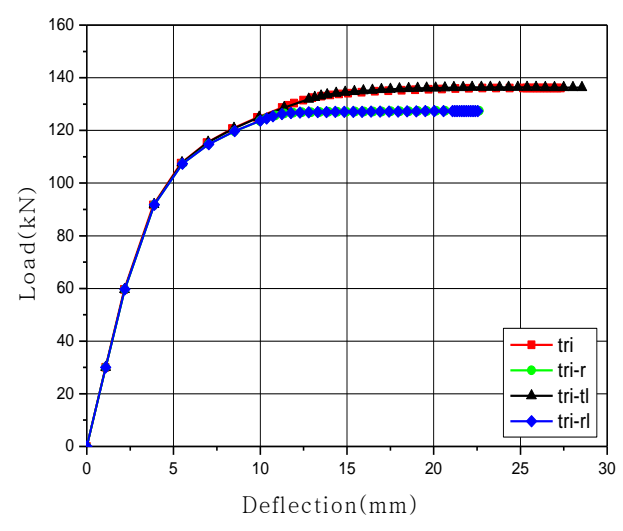

Fig. 5. Load-deflection curve of front tri model。 
When inner frame is triangular frame (rec-t) or rectangular frame (rec-r), load-deformation curve was similar but the maximum load capacity of triangular frame was higher than rectangular frame by $2 \%$. When an inner frame is an extended triangular frame (rec-tl) or an extended rectangular frame (rec-rl), maximum load capacity was reduced by $15 \%$ due to sudden buckling because when outer frame is in a rectangular frame, binding force of the joint is caused by horizontal sub-braces of outer frame and thus rapid reduction in load capacity was occurred after buckling.

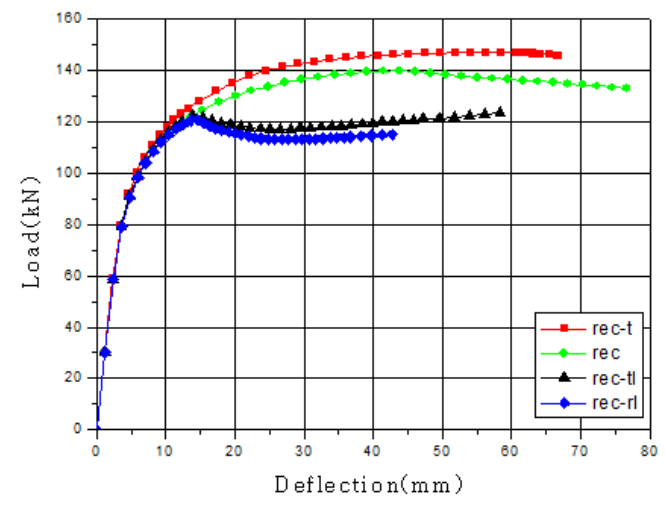

Fig. 6. Load-deflection curve of front rec model

\section{5) Outer extended triangular frame}

Fig.7 shows the graph comparing the load-deformation curve with an outer frame in the type of extended triangular frame (tri-long) and 4 types of inner frame such as tri, rec, tri-long and rec-long. As seen in the graph, initial stiffness of 4 frames was similar each other but when the inner frame is an extended triangular frame (tri-tl,tri-rl), maximum load capacity was reduced.

Load-deformation curve when the inner frame was a triangular frame (tri-long-t) and rectangular frame (tri-long-r) was similar each other which was attributable to in-plane buckling occurred on outer extended frame, irrespective of the shape of inner frame. In case of triangular frame (trilong-tl) or rectangular frame (rec-long-rl) with extended inner frame, maximum load capacity was reduced by $10 \%$ which was attributable to out-of-plane buckling on outer frame when the load capacity was less than in-plane buckling load capacity.

\section{6) Outer extended rectangular frame}

Fig. 8 shows the graph for comparing with outer frame in the type of extended rectangular frame (rec-long) while inner frame is classified into t types such as tri, rec, tri-long and rec-long. As seen in the graph, initial stiffness of 4 frames was similar each other but the maximum load capacity was higher only when inner frame was in the type of triangular frame.

Out-of-plane buckling was occurred to all of 4 types and the case of inner frame in type of triangular frame, unlike other 3 cases, was attributable to axial force occurred on horizontal sub-braces of inner frame which resulted in constraining the movement of the joint in out-of-plane direction.

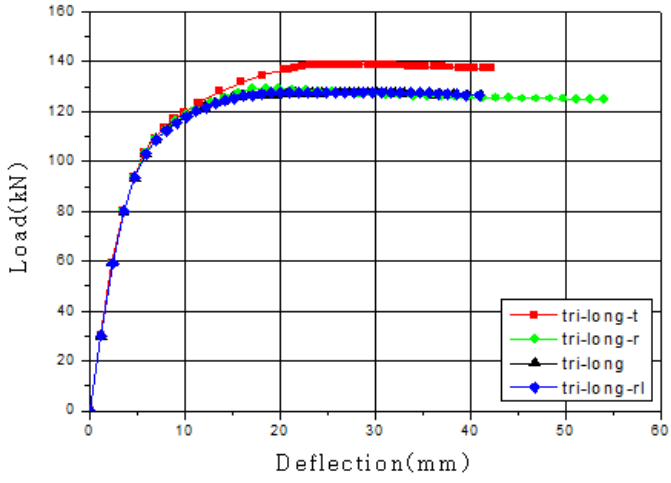

Fig. 7. Load-deflection curve of Fronttri-long model.

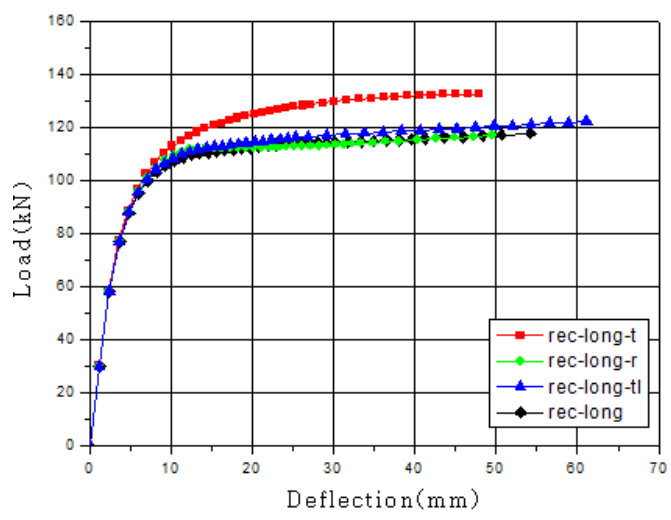

Fig. 8. Load-deflection curve of front rec-long model.

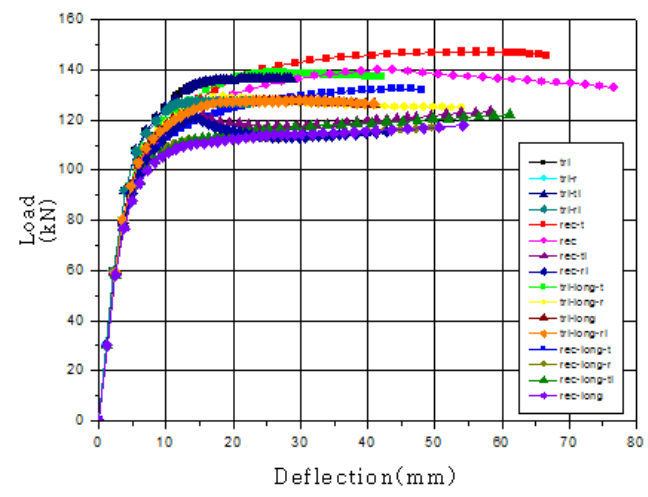

Fig. 9. Load-deflection curve of all models.

TABLE IV: COMPARISON OF MAXIMUM LOAD CAPACITY AND DISPLACEMENT

\begin{tabular}{|c|c|c|}
\hline model name & $\begin{array}{c}\text { Maximum load } \\
\text { capacity } \\
(\mathrm{kN})\end{array}$ & $\begin{array}{c}\text { Displacement at } \\
\text { max load } \\
(\mathrm{mm})\end{array}$ \\
\hline Tri-t & 136 & 25.9 \\
\hline Tri-r & 136 & 23.2 \\
\hline Tri-tl & 127 & 22.6 \\
\hline Tri-rl & 127 & 22.5 \\
\hline Rec-t & 147 & 56.2 \\
\hline Rec-r & 139 & 43.7 \\
\hline Rec-tl & 122 & 13.9 \\
\hline
\end{tabular}




\begin{tabular}{|c|c|c|}
\hline Rec-rl & 120 & 14.5 \\
\hline Tri-long-t & 139 & 25.8 \\
\hline Tri-long-r & 129 & 18.5 \\
\hline Tri-long-tl & 127 & 28.2 \\
\hline Tri-long-rl & 127 & 29.7 \\
\hline Rec-long-t & 132 & 45.0 \\
\hline Rec-long-r & 116 & 49.5 \\
\hline Rec-long-tl & 122 & 61.1 \\
\hline Rec-long-rl & 117 & 54.2 \\
\hline
\end{tabular}

\section{Comparison of Maximum Load Capacity}

As aforementioned, maximum load capacity and displacement obtained as a result of combining the outer frame and inner frame in different shapes are summarized in Table IV. and Fig.9. Maximum load capacity was generally $116 \mathrm{kN}$ to $147 \mathrm{kN}$ and the displacement under maximum load capacity was $13.9 \mathrm{~mm}$ to $61.1 \mathrm{~mm}$. Load capacity of the frame was relatively more dependent on inner frame shape than outer frame. In-plane bucking occurred when inner frame was in type of triangular frame while out-of-plane buckling occurred when inner frame was rectangular frame, resulting in reduction in buckling load capacity. Thus leg frame load capacity was more dependent on inner frame than outer frame and load capacity of outer frame remained unchanged even in case of rectangular frame.

\section{CONCLUSION}

In this study, the role of the sub-braces and behavioral characteristics of the frame were identified by means of 3D nonlinear analysis and comparative verification of transmission tower so as to come up with the solution to minimize the sub-braces as well as to provide the basic data to simplify the frame shape of existing transmission tower. The effect of sub-braces on the tower was evaluated in a way of combining the frames variously while varying the shape of outer frame and inner frame depending on availability of the sub-braces and as a result, following conclusion was made.
1) $2 \mathrm{D}$ analysis is adopted for current transmission tower design without considering the out-of-plane buckling. But the result of finite element analysis in this study showed buckling mode occurred in out-of-plane direction. Thus 3D analysis of buckling behavior is necessary.

2) As a result of comparing and analyzing the structural behavior of triangular frame and rectangular frame, failure of triangular frame occurred by partial buckling on member, while the failure of rectangular frame occurred by overall buckling on member. But the maximum load capacity and deformation is similar in both cases.

3) As a result of comparing and reviewing the maximum load capacity by combining the outer frame and inner frame for LEG, usability of rectangular frame was demonstrated.

4) The sub-braces are able to increase the buckling load capacity by reducing the buckling length, but excessive subbraces are not able to improve the load capacity of the transmission and moreover it may increase the weight of the member which rather reduces the cost efficiency and work efficiency. Thus, it's necessary to determine the use of subbraces after reviewing in depth the in-plane and out-of-plane buckling behavior.

\section{ACKNOWLEDGEMENT}

This research was supported by Korea Electric Power Corporation. (Grant number:R17XA05-24)

\section{REFERENCES}

[1] Korea Electric Power Company, Specification of Electric Transmission Tower 1111,1997

[2] F. G. A. Al-Bermani and S. Kitipornchai, "Nonlinear analysis of transmission towers," Eng.Struct., vol. 14, no. 3, 1992, pp.139-151

[3] W. B. Kim, "Development of nonlinear analysis technic to determine the ultimate load in electric transmission tower," International Journal of Steel Structure, vol.13, no. 2, pp. 305-315,June,2013

[4] S. L. Chan and S. Kitipornchai, "Inelastic post-buckling behavior of tubular struts," ASCE, Journal of Structural Engineering, vol. 114, no. 5, 1998, pp.1091-1104

[5] S. Roy, "Secondary stresses on transmission tower structures," ASCE, Journal of Structural Engineering, vol. 110, no. 2,1984, pp.157-172

[6] Bleich, Buckling Strength of Metal Structures, McGraw-Hill,1952, pp. $20-30$

[7] F. Albermani, "Upgrading of transmission towers using a diaphram bracing system," Engineering Structures, vol. 26, 2004.

[8] ABAQUS User's Manual Version 6.5, Hibbit Karlsson, and Sorensen, Inc.,Pawtucke, R,I.

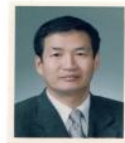

2012 2014
Woo Bum Kim was born in Seoul, Korea, december,1956 $\mathrm{Ph} . \mathrm{D}$, Civil Engineering, Lehigh University, Pennsylvania, U.S.A, 1992, (structural engineering)Now, he is working as professor at the dept. of Architectural engineering in Kongju National University, KOREA. Professor Kim engaged in Korean Society of Steel Construction as vice president from 